\title{
Conceptions in Making Sense of Multiplication
}

\author{
Kin Eng Chin ${ }^{1 *}$, Fui Fong Jiew ${ }^{2}$, Elvent Taliban ${ }^{3}$ \\ ${ }^{1}$ Flinders University, AUSTRALIA \\ ${ }^{2}$ Queensland University of Technology, AUSTRALIA \\ ${ }^{3}$ Universiti Malaysia Sabah, MALAYSIA
}

Received 30 November 2018 - Revised 8 March 2019 - Accepted 9 March 2019

\begin{abstract}
This paper aims to explore how three secondary school teachers make sense of multiplication based on their conceptions of multiplication. The framework of supportive and problematic conceptions in making sense of mathematics proposed by Chin (2013) is exemplified in this study. Initially, data were collected through a survey assessment that consists of a set of mathematical tasks. Then follow-up interviews were conducted with these participants. Three secondary school teachers (Jessica, Sarah and Claire - pseudonyms) participated in this study. Findings revealed that the changes of meanings in multiplication have impacted the sense-making of Sarah and Claire. On the other hand, Jessica who sensed these mathematical changes could talk about multiplication sensibly.
\end{abstract}

Keywords: changes of meanings, multiplication, supportive conceptions, problematic conceptions, sense-making

\section{INTRODUCTION}

It is undeniable that one of the ultimate aims of mathematics education is to help learners to make sense of mathematics. Learning mathematics without sense making may end up as rote learning. This sparks an interesting question, what is sense making in mathematics? According to the respected USA National Council of Teachers of Mathematics (NCTM, 2009), "sense making may be considered as developing understanding of a situation, context, or concept by connecting it with existing knowledge or previous experience" (p.4). When mathematics educators introduce new concepts, they must link new concepts with pupils' existing knowledge or prior experience so that they can make sense of mathematics. But how do mathematics educators help students to link new concepts with existing knowledge or prior experience? Mathematics educators need to understand the underlying mechanism on how humans link these constructs together so that they can provide sensible mathematics to pupils. This study attempts to illuminate the underlying mechanism for mathematics sense making by interviewing three secondary school teachers after they have answered a set of mathematical tasks related to multiplication. We believe that teachers need to be able to make sense of mathematics that they are going to teach then only they can help their pupils to make sense of the mathematics. Besides that, mathematics educators also need to have a good grasp of the different aspects of mathematical concepts. This will enable them to identify those aspects that are supportive to build on and also those problematic aspects that need to be addressed explicitly in order to avoid misconceptions.

\section{RESEARCH STUDIES OF MULTIPLICATION}

In schools, teaching of mathematics recurs on drilling, memorising facts and procedures (Abdullah, Halim \& Zakaria, 2014). These are essential in learning mathematics. However, memorising facts without an awareness of changes of mathematical meanings and executing procedures without sense-making may result in rote learning. As a result, learners may not be able to solve non-routine problems. Hence, teaching multiplication requires mathematics teachers to understand how the multiplication algorithm works before they can identify appropriate learning opportunities for students (Whitacre \& Nickerson, 2016). The findings in Saleh, Saleh, Rahman and Mohamed (2010) revealed that most of the 202 Year Two primary school pupils under studied can solve standard

(C) 2019 by the authors; licensee Modestum Ltd., UK. This article is an open access article distributed under the terms and conditions of the Creative Commons Attribution License (http://creativecommons.org/licenses/by/4.0/). 《kineng.chin@flinders.edu.au (*Correspondence) $\square$ fuifong.jiew@hdr.qut.edu.au $\$ elitesquad89@gmail.com 


\section{Contribution of this paper to the literature}

- This study fills in a research gap that focuses on changes of mathematical meanings for multiplication in daily life contexts that may support or impede the sense making of learners.

- Findings indicate that changes of meanings in mathematics present great difficulties to teachers who are not aware of those changes.

- This paper exemplifies a potentially useful framework which is known as supportive and problematic conceptions that may be regarded as a useful tool for mathematics education community to understand the sense making process of learners in mathematics.

operation for multiplication based on multiplication tables and multiplication facts but this didn't help them to solve real life problems. On the other hand, Southwell and Penglase (2005) found that prospective elementary teachers can solve multiplication problems but they can't explain why it works. In another study done by Harkness and Thomas (2008), the participants tended to put emphasis on the steps in alternative multiplication algorithm and they also didn't realise why it works. Thus, mathematics teachers need to reason sensibly and flexibly while doing mathematics (Whitacre \& Nickerson, 2016) so that students can understand mathematical concepts through understanding the underlying meanings of mathematical operations (Burris, 2005).

Making sense of multiplication is a complex process (Chin \& Jiew, 2019). A mathematics expert teacher in the study of Jiew and Chin (in press) reasoned inaccurately when he was required to explain the meaning of multiplication across different contexts. His initial interpretation of multiplication as repeated addition has restricted him to explain sensibly when the multiplier was a negative integer. Larsson, Pettersson and Andrews (2017) stated that multiplication which is introduced exclusively as repeated addition or equal groups is a problematic instruction and Vosniadou and Verschaffel (2004) stated that this problematic instruction may create unhelpful synthetic conceptions when the instruction given is building from previous knowledge. Findings of Larsson, Pettersson and Andrews (2017) revealed that the way teachers taught multiplication as repeated addition was problematic, especially encountered with multiplication of multi-digits and decimals. In fact, there are other researchers who also argued that solving multiplication problems solely based on repeated addition is not enough (Byers, 2007; Devlin, 2007). In the similar vein, Chin and Jiew (2019) argued that it is possible to have other meanings of multiplication thus they explored the conceptions of two mathematics teachers possessed about the multiplication symbol $(\times)$ in order to look for some possible alternative conceptions. The teachers were required to give real life examples based on the given mathematical expressions i.e. from symbolism to a real life context. The findings revealed that multiplication may be interpreted as repeated subtraction when the multiplier is a negative integer and this is consistent with Kilham (2011) who also stated that a positive multiplier means an iterated addition and a negative multiplier means an iterated subtraction.

This study is different from Chin and Jiew (2019) where participants are required to create mathematical expressions based on the given real life problems i.e. from different real life contexts to symbolism. We feel that it would be fruitful to explore how participants communicate their mathematical ideas happen in real life into mathematical symbols.

\section{THEORETICAL FRAMEWORK}

Mathematical concepts are multifaceted. They have different meanings in different contexts (Chin \& Pierce, 2019). Chin (2013) and Chin and Jiew (2018) have formulated a framework that highlighted how the changes of meanings in mathematical concepts affect the sense making of humans. This framework is known as the supportive and problematic conceptions. In this case, supportive conceptions refer to conceptions that work in an old context and continue to work in a new context. In contrast, problematic conceptions refer to conceptions that work in an old context but do not continue to work in a new context. Take for instance, the conception of adding two numbers will yield a bigger number. This conception might develop when learners work in the context of whole numbers. When learners move to the context of positive integers then this conception still valid. Thus this conception that originates from whole numbers context may be regarded as a supportive conception in the context of positive integers. However, when learners move to the context of negative integers then the conception of adding two numbers will yield a bigger number is not valid anymore. Thus this conception may be regarded as a problematic conception in the context of negative numbers.

Based on Skemp (1971), "To understand something means to assimilate it into an appropriate schema" (p.46). In this case, schemas are the building blocks of knowledge. The framework of this study is consistent with the notions of assimilation and accommodation of schema as proposed by Piaget (1952). When the learning of new concepts fits with existing knowledge schema then assimilation may occur smoothly. This resonates with the idea of supportive conceptions. Bear in mind that human conceptions could be developed through prior learning and 
they contribute to the formation of schema. Hence, supportive conceptions occur when the conceptions that arise from previous learning are consistent with new learning. On the other hand, when the learning of new concepts contradicts with existing knowledge schema then accommodation of schema needs to be performed in order to progress. This is similar to a situation where problematic conceptions that arise from previous learning conflict with new learning. The framework of supportive and problematic conceptions is a powerful framework because it offers mathematics educators more insights on how assimilation and accommodation occur. In addition to that, it leads us to see clearly what conceptions or aspects that support or impede the development of a coherent schema. As an illustration, $2 \times 3$ may be regarded as 2 lots of 3 and this may be interpreted as $3+3$ which is equal to 6 and this could be conceived as repeated addition. In the context of measurement, take for instance, $(2$ kilometers per hour) $\times 3$ hour then the product of speed times time is distance and the answer is 6 kilometers. The operational aspect of these two instances is similar however the meaning is different. The framework of supportive and problematic conceptions is employed to guide the development of the mathematical tasks with an aim to elicit participants' supportive and problematic conceptions in solving the given problems that in turn could demonstrate how these personal conceptions influence the sense making of the participants.

In this study, the mathematical concept that we want to focus is multiplication because this is one of the fundamental mathematical operations that has a very wide application in solving various types of problems. Based on the collected data, we aim to discern how participants use multiplication in different contexts. All the tasks may be solved by using multiplication only and they present a range of contexts. The variance of contexts with an invariant solving strategy can lead us to discover the changes of mathematical meanings across a variety of contexts.

\section{METHODOLOGY}

According to Chin (2013) and Chin and Jiew (2018), mathematical concepts and symbols change meanings according to different contexts. Therefore we conjecture that the changes of mathematical meanings are a barrier in making sense of multiplication. This difficulty is not limited to pupils only and we speculate that mathematics teachers may face this difficulty as well. Teachers and students need to deal with mathematics in a variety of contexts thus it is important for them to be able to make sense of multi-contextual mathematics

This is a preliminary study for a bigger project that aims to investigate how humans make sense of mathematics over the longer term. In other words, it is to elicit participants' conceptions regarding the meanings of multiplication across different contexts. Specifically, this preliminary qualitative study aims to answer the following research questions:

1. What are the supportive and problematic conceptions related to multiplication in solving daily life problems?

2. How do the participants react to their supportive and problematic conceptions of multiplication?

3. Are the participants aware of the changes of mathematical meanings of multiplication based on the daily life contexts given?

We decided to have mathematics teachers as our participants for this study. This is because teachers need to be able to make sense of mathematics so that they can help their students in understanding mathematics. Thus exploring how mathematics teachers make sense of mathematics is important for the mathematics education community. Initially an invitation was sent to a group of potential teachers. There were twenty teachers in this group. All the potential teachers possess at least a year of mathematics teaching experience and an academic qualification that is related to mathematics. Three participants (Jessica, Sarah and Claire - pseudonyms) responded to our invitation and participated this study on voluntary basis. Jessica and Sarah were secondary school teachers who possessed an education degree with a major in physics and a minor in mathematics. Jessica had ten years of teaching experience whereas Sarah had 8 years. On the other hand, Claire possessed an education degree with a major in mathematics and a minor in commerce. She had one year of teaching experience. All of them taught mathematics and other subjects.

We used a phenomenological approach to collect data, a process that was performed in two steps: a survey assessment and a follow-up interview. The survey assessment consists of a set of mathematical tasks with seven items with an intention to elicit supportive and problematic conceptions of the participants. These items were adapted from the Malaysia Mathematics textbooks-Chan and Puteh (2016), Ooi, Yong and Ng (2017) and Baharam, Baharam, Ahmad, Tahir and Hanafiah (2017). Since these participants were mathematics teachers thus they must have encountered these types of questions before. The participants responded to the given mathematical tasks first then follow-up interviews were conducted with each of them to gain further insights on their thinking. The intent of this research design is to provide a platform for the participants to reflect on their working for solving the given mathematical tasks. These reflections were externalized by asking the participants to reason their chosen mathematical procedures and symbols during the follow-up interviews. This study extends the work of Chin and Jiew (2019) that was focusing on eliciting participants' conceptions of multiplication by using mathematical 


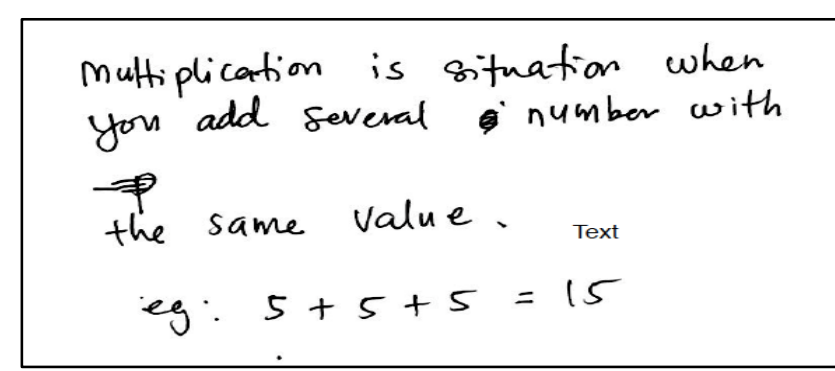

Figure 1. Jessica's response to initial conception for multiplication

expressions. In this study, we used mathematics word problems to elicit participants' conceptions of multiplication without providing mathematical expressions in the items so that we can investigate whether the participants were aware of the changes of mathematical meanings or not. In this study, we have chosen multiplication $(\times)$ as our topic of investigation. In general the participants only took about 15 minutes to complete all the items. The first item required the participants to describe multiplication in their own words. This item aimed to explore how the participants interpret multiplication at the beginning of the study. The remaining six items were about different real life problems and the participants were required to solve them. The purpose of using these mathematical tasks is to explore how the participants solve these tasks. Then follow-up interviews were conducted with each of the participants individually in order to capture the reasoning process behind their written work. In this paper, qualitative differences of the collected responses will be presented by reporting the data collected from 5 items so as to highlight how supportive and problematic conceptions affect the sense making of the participants.

\section{RESULTS}

In order to answer the proposed research questions of this study, relevant data were extracted from the mathematical tasks and follow-up interviews in order to highlight the qualitative differences between these teachers in reasoning the performed operation for getting the solutions. We explored the initial conceptions of these teachers by asking them to describe multiplication in their own words. In general, all the participants interpreted multiplication as repeated addition. Jessica provided a typical written response.

To the first item of the survey assessment "Mark had 2 bags of stars. There are 3 stars in each bag. How many stars does Mark have altogether?" All the participants got the correct answer as 6 stars by using multiplication and they could elaborate how multiplication was related to repeated addition. Claire, for example, stated in the follow up interview that

\section{It is just the same number that is repeatedly being added to form a new number (pointing at her writing $3+3)$.}

This indicates that the participants were able to see how multiplication was related to repeated addition and they could either use $2 \times 3$ or $3+3$ to get the answer. At this particular instance, the participants' initial conception of multiplication as repeated addition was applicable to this real life problem.

The second item was related to a problem which involved money. Item 2 of the survey assessment said "The cost of a pen is 2 dollars. Peter bought 4 pens. How much did he pay for the pens? Again, all the teachers got the correct answer by using multiplication. All the three teachers managed to link multiplication to repeated addition in this case. They had a coherent understanding for multiplication. This showed that repeated addition was a supportive conception in this context. In the follow-up interview, Claire further elaborated that

\section{Actually number two is added repeatedly four times $2+2+2+2$ and then I just used multiplication so it will be easier.}

Item 4 was a problem related to measurement. This kind of problem is common in engineering sectors. Item 4 asked "A rectangular playground is 8 meters wide and 12 meters long. What is the area of the playground?" All the teachers got the correct answer however not all of them could make sense of the performed procedures. During the follow-up interviews, we asked the teachers to explain why they used multiplication to solve this question. The following interview excerpts show their explanations.

\footnotetext{
The formula of area is length times width so I used multiplication... I think it is not repeated addition because I will not get the same answer if I used repeated addition...hmmm(feeling sceptical)...eight eight sixteen (mumbling then she used a calculator to test)... yes, for this one it is still repeated addition. [Claire]
} 


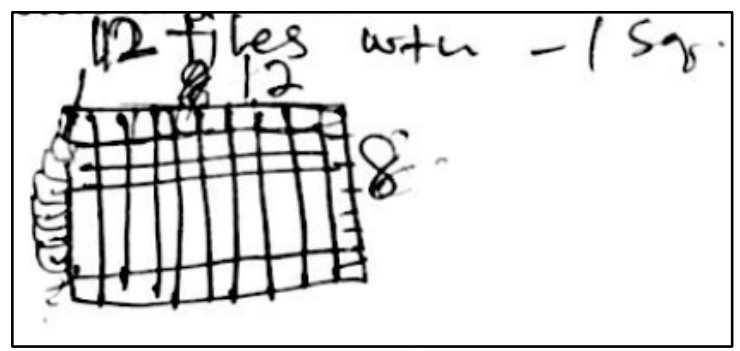

Figure 2. Grid diagram by Jessica

I used multiplication because of the area formula which is length times width...I think it is still repeated addition...ermm...but if we explain to students, I think there will be some confusion if we say that calculating area is repeated addition. For example, the number 8 is added repeatedly 12 times and the result is the same as the answer for this question...the formula itself already indicated multiplication...maybe if someone feels that multiplication is hard, so maybe they can repeatedly adding 8 for 12 times... better straight away follow the formula.

[Sarah]

Formula for finding the area of rectangle is width times length so 8 times $12 \ldots$ It is repeated addition... (Drawing a grid (see Figure 2)) If I imagine this playground with tiles on it and the tiles are one unit meter long and one unit meter wide so if we use repeated addition then the total number of tiles inside the playground is 96 tiles with 1 square meter each.

[Jessica]

Based on the above excerpts, it can be noticed that Claire couldn't link repeated addition with multiplication in a meaningful way. Initially she felt that multiplication was not repeated addition for this case because she couldn't find a way to link them together. Then she used a calculator to verify her answer by repeatedly adding 8 for 12 times. By using the calculator, she got an answer which was the same answer as calculated by using the area formula. Then she changed her interpretation by saying that multiplication was repeated addition in this case because she could get the same answer by using repeated addition. The issue was she couldn't make sense of the area formula meaningfully. If she could derive the area formula then she might probably see how repeated addition is related to multiplication at this particular task. This showed that Claire perceived repeated addition as a supportive conception at this particular instance due to the fact that she could get the correct answer by using repeated addition. Obviously the operational aspect of multiplication was supportive. However, there is a change of meaning in this new context and Claire was not aware of it thus she couldn't make sense of why multiplication could be used in this case because she was thinking purely in the context of natural numbers.

Sarah faced the same issue as Claire. She couldn't link multiplication with repeated addition meaningfully and she did a similar thing like Claire by checking the final answer produced by the area formula through repeated addition. This sparks an interesting question of why Sarah and Claire couldn't link multiplication with repeated addition at this particular context? This is probably because there is a change of meaning for multiplication due to a change of context. When two units of length measurement multiply together, an area is produced. For instance, 8 meters multiply 12 meters will equal to 96 square meters. Both Sarah and Claire focused on the final answer of this problem. When the final answer could be obtained by repeated addition besides multiplication then they claimed that multiplication was repeated addition. However, this didn't help them to make sense of multiplication successfully.

Jessica was able to sense the change of meaning for this problem by using a grid diagram. She realized that the area of the playground had to be divided into small squares with same size and the area of each square was 1 square meter. She imagined these small squares as tiles on the playground. Then she could add these tiles together to get the total area of the playground. Based on this diagram, she could visualize how repeated addition was related to multiplication. Thus she did build a coherent understanding for multiplication.

Item 6 of the survey assessment said " $\frac{3}{4}$ of a pan of brownies was sitting on the counter. You decided to eat $\frac{1}{3}$ of the brownies in the pan. How much of the whole pan of brownies did you eat?" Jessica and Sarah got the correct answer as $\frac{1}{4}$ by using multiplication but Claire got the incorrect answer for this item by using subtraction. The reasons for choosing the selected procedure to solve this problem were explored in the individual follow-up interviews.

Based on the question "because I decided to eat 1 over 3" so it means subtraction (pointing to her written answer, see Figure 3) [Claire] 


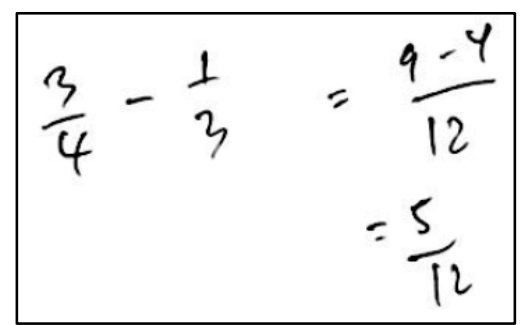

Figure 3. Claire's response to Item 6 I still use multiplication...ermmmm...I cannot see where is the repeated addition because 3 over
4 ...ermmm...I don't know how to find (laughing), but maybe there is still repeated addition
but...because I don't know how many times to add for 3 over 4 so here I can only see the multiplication
process...I am unable to relate it back to my description of multiplication.
[Sarah]

Because I recall using a formula... a mathematics formula if we want to find a value from a certain part of a cake or a fraction, we can just multiply... I think I cannot recommend to use repeated addition with this question...I think multiplication has its own circumstances...some situations can use repeated addition and some cannot.

Claire used subtraction to get her answer which was incorrect. We all know that subtraction may be interpreted as taking away something. Claire noticed the word "eat" and that was equivalent to "taking away" thus she used subtraction to solve this problem. Sarah used multiplication to solve item 6 but she couldn't give a particular reason of why she could use this operation. It might be due to her past experience of solving similar problems. She was trying to make sense through repeated addition but that was unsuccessful. She wasn't aware the change of meaning in this context. On the other hand, Jessica did sense the change of meaning in this problem and she realized that she couldn't make sense of this situation by using repeated addition. Additionally, she was aware that multiplication cannot be interpreted as repeated addition in all the situations.

\section{DISCUSSION}

Mathematics teachers need to deal with mathematics in a variety of contexts. The implication is teachers have to be aware with the changes of meanings due to changes of contexts. If teachers couldn't sense the changes of meanings then they will end up with procedural teaching and forcing students to memorize and use the formulae without a real understanding. It can be noticed that the interpretation of repeated addition was a supportive conception for first and second items (i.e. the stars problem and the pens problem) for all the participants. Then this interpretation became a problematic conception for items 4 and 6 (i.e. the playground problem and the brownies problem) in particular for Sarah and Claire. Even though the area formula can be linked to repeated addition, it is undeniable that there is still a change of meaning where multiplying lengths gives an area. We can see that Sarah and Claire who didn't aware of the changes of mathematical meanings eventually ended up with using formulae that they couldn't make sense of for the playground problem. They knew one of the interpretations for multiplication that was repeated addition. When the meaning of multiplication changes then they rote learned the procedure/formulae.

On the other hand, Jessica who was aware of the changes of mathematical meanings managed to provide sensible explanations for her working. Based on the data analysis, we can see that how the changes of mathematical meanings have impeded the sense making of the participants. Based on the collected data, it can be noticed that problematic conceptions impede sense making whereas supportive conceptions support generalization. Hence, when humans encounter supportive conceptions, they may assimilate them into a schema easily and help them to make sense of mathematics. On the contrary, humans need to accommodate their schema when encounter problematic conceptions so that they can progress. If learners are not aware of the problematic conceptions then they may end up being a rote learner without making sense of the mathematics. The bigger picture is teachers need to be sensitive with the changes of mathematical meanings in multi-contextual mathematics so that they can guide their students to make sense of mathematics.

\section{CONCLUSION}

In conclusion, repeated addition may be considered as a supportive conception for the first two items (i.e. the stars problem and the pens problem). Then it gradually became a problematic conception for the next two items. Sarah and Claire were not aware of the changes of meanings of multiplication and this has greatly affected their 
sense making. In contrast, Jessica who sensed the changes of meanings managed to link ideas in a consistent way based on different contexts and this equipped her with the ability to provide sensible explanations. This indicated that Jessica was able to accommodate her schema in dealing with the given multi-contextual mathematics. This study is of high significance due to the point that the constructs of supportive and problematic conceptions are demonstrated to be useful in helping us to understand how assimilation and accommodation occur in the human mind. On top of that, this framework also offers us with more details on what are the aspects that are supportive or problematic in the sense making process. Humans need to be aware of changes of mathematical meanings in order to make sense of mathematics (Chin, 2013).

\section{REFERENCES}

Abdullah, N., Halim, L., \& Zakaria, E. (2014). VStops: a thinking strategy and visual representation approach in mathematical word problem solving toward enhancing stem literacy. EURASIA Journal of Mathematics, Science \& Technology Education, 10(3), 165-174.

Baharam, B. H., Baharam, B. H., Ahmad, N. J., Tahir, N. M., \& Hanafiah, M. N. M. (2017). Matematik tingkatan 2.

Burris, A. C. (2005). Understanding the Math You Teach Content and Methods for Prekindergarten Through Grade 4.

Byers, W. (2007). How mathematicians think: using ambiguity, contradiction, and paradox to create mathematics.

Chan, Y. L., \& Puteh, M. (2016). Matematik tahun 6 sekolah kebangsaan.

Chin, K. E. (2013). Making sense of mathematics: supportive and problematic conceptions with special reference to trigonometry (Unpublished doctoral thesis). Retrieved from http:/ / wrap.warwick.ac.uk/58403/

Chin, K. E., \& Jiew, F. F. (2018). A framework for making sense of mathematics. In F. J. Hsieh (Ed.), Proceedings of the 8th ICMI-East Asia Regional Conference on Mathematics Education, Vol. 2, (pp. 309-319).

Chin, K. E., \& Jiew, F. F. (2019). Changes of meanings in multiplication across different contexts: The case of Amy and Beth. EURASIA Journal of Mathematics, Science and Technology Education, 15(8), em1739. https://doi.org/10.29333/ejmste/108440

Chin, K. E., \& Pierce, R. (2019). University students' conceptions of mathematical symbols and expressions. EURASIA Journal of Mathematics, Science and Technology Education, 15(9), em1748. https://doi.org/10.29333/ejmste/103736

Devlin, K. (2007). What is conceptual understanding? Retrieved from http://www.maa.org/external_archive/devlin/devlin_09_07.html

Harkness, S. S., \& Thomas, J. (2008). Reflections on "Multiplication as original sin": The implications of using a case to help preservice teachers understand invented algorithms. Journal of Mathematical Behavior, 27(2), 128-137. https:/ / doi.org/10.1016/j.jmathb.2008.07.004

Jiew, F. F., \& Chin, K. E. (in press). Supportive and problematic conceptions in making sense of multiplication: A case study. The Mathematics Enthusiast.

Kilhamn, C. (2011). Making sense of negative numbers (Doctoral thesis). Retrieved from https:/ / gupea.ub.gu.se/handle/2077/24151

Larsson, K., Pettersson, K., \& Andrews, P. (2017). Students' conceptualisations of multiplication as repeated addition or equal groups in relation to multi-digit and decimal numbers. The Journal of Mathematical Behavior, 48, 1-13. https:// doi.org/10.1016/j.jmathb.2017.07.003

National Council of Teachers of Mathematics (2009). Focus in High School Mathematics: Reasoning and Sense Making. Retrieved from https:/ / www.nctm.org/Standards-and-Positions/Focus-in-High-School-Mathematics /

Ooi, S. H., Yong, K. Y., \& Ng, S. H. (2017). Matematik tingkatan 1.

Piaget, J. (1952). The origins of intelligence in children (M. Cook, Trans.). https:/ / doi.org/10.1037/11494-000

Saleh, S., Saleh, F., Rahman, S. A., \& Mohamed, A. R. (2010). Diagnosing year two pupils understanding of the certain concepts of multiplication at selected schools in Sabah. Procedia Social and Behavioral Sciences (Elsevier Ltd.), 8, 114-120. https:/ / doi.org/10.1016/j.sbspro.2010.12.016

Skemp, R. (1971). The Psychology of Learning Mathematics.

Southwell, B., \& Penglase, M. (2005). Mathematical knowledge of pre-service primary teachers. In H. L. Chick, \& J. L. Vincent (Eds.), Proceedings of the 29th Conference of the International Group for the Psychology of Mathematics Education, 4, 209. 
Vosniadou, S., \& Verschaffel, L. (2004). Extending the conceptual change approach to mathematics learning and teaching. Learning and Instruction, 14(5), 445-451. https:/ / doi.org/10.1016/j.learninstruc.2004.06.014

Whitacre, I., \& Nickerson, S. D. (2016). Investigating the improvement of prospective elementary teachers' number sense in reasoning about fraction magnitude. Journal of Mathematics Teacher Education, 19(1), 57-77. https:/ / doi.org/10.1007/s10857-014-9295-2

\section{http://www.ejmste.com}

